\title{
10 Molières Komödie über das aufrichtige Sprechen: Le Misanthrope
}

Der unbestrittene Höhepunkt der literarischen Reflexion über die Form der indirekten Kommunikation im 17. Jahrhunderts ist Molières Komödie Le Misanthrope (1666). Indirektes Sprechen ist das Thema des Stücks. Der Protagonist der Komödie, Alceste, sucht ein Leben jenseits der Heuchelei zu führen. Sein Ideal ist die Wahrhaftigkeit. Zwischen ihm, seinem Freund Philinte, der von ihm verehrten Célimène sowie den weiteren Personen des Stücks entspinnt sich über den gesamten Verlauf der Komödie eine Diskussion über die Notwendigkeit der Anpassung an gesellschaftliche Normen und speziell über die Frage, ob man sich den Regeln der zeitgenössischen Kommunikation bei Hof und in den Salons unterwerfen soll. Am Ende des Stücks erklärt Alceste, dass er sich nun endgültig aus der Gesellschaft zurückzuziehen gedenkt. In Molières Komödie werden durch die Diskussionen und Reflexionen grundlegende Wertvorstellungen des ,honnnête homme، einer Prüfung unterzogen: Ist die Wahrhaftigkeit des Alceste ein Verstoß gegen die ,bienséance‘ und den guten Geschmack? Sind die Forderungen der ,politesse und ,bienséance‘ ethisch-moralischer, ästhetischer Natur oder Zwänge, den Einzelnen gesellschaftlichen Normen zu unterwerfen? Verkörpert Alceste eher jene Anschauungen der alten schwertadeligen Territorialfürsten, die der Gradlinigkeit und Aufrichtigkeit verpflichtet sind, oder bereits bürgerliche Vorstellungen von Ehrlichkeit, über die sich das Bürgertum vom Adel abzugrenzen sucht? Auch der Misanthrope hat eine prominente Rezeptionsgeschichte: Nachdem Rousseau kritisiert, dass Molière seinen Helden Alceste dem Gespött der Zuschauer preisgibt, verfasst Jean-François Marmontel eine moralische Erzählung mit dem Titel: Le Misanthrope corrigé (1765), die von Charles-Albert Demoustier als Komödie Alceste à la campagne, ou le Misanthrope corrigé 1790 in eine Bühnenfassung übersetzt wird ${ }^{1}$. Im gleichen Jahr schreibt einer der Protagonisten der Französischen Revolution, Philippe-François-Nazaire Fabre, genannt Fabre d'Eglantine, eine von ihm als Fortsetzung deklarierte Version des Stücks: Philinte, ou la suite du Misanthrope $\left(1790^{2}\right)$.

1 Vgl. dazu Joseph Harris, Marmontel and Demoustier, Le Misanthrope corrigé. Two EighteenthCentury Sequels to Molière's Le Misanthrope (Modern Humanities Research Association. Critical Texts. 65), Cambridge, The Modern Humanities Research Asscociation, 2019.

2 Vgl. dazu Jürgen von Stackelberg, „Zu Fabre d'Eglantines Le Philinthe de Molière“, Romanische Forschungen Bd. 97/1985, S. 390-401, sowie Ronald W. Tobin, „Le Misanthrope revu et corrigé: Le Philinte de Fabre d'Églantine“, in: Martial Poirson (Hrsg.), Ombres de Molière. Naissance d'un mythe littéraire à travers ses avantars du XVIIe siècle à nos jours, Paris, Colin, 2012, S. 367-380.

2 Open Access. (c) 2021 Michael Bernsen, publiziert von De Gruyter (c))BY-NC-ND Dieses Werk ist lizenziert unter der Creative Commons Attribution 4.0 Lizenz.

https://doi.org/10.1515/9783110758009-010 
Die Forschung über Molières Misanthrope hat sich naturgemäß zumeist auf die Frage konzentriert, mit welchen Verfahren welche Art von Komik im Stück erzeugt wird. Dass der Held der Komödie als ,atrabilaire amoureux“, wie es im Untertitel heißt, also als acharnierter Melancholiker und zugleich als Verliebter, somit als ein widersprüchlicher Charakter, angelegt ist, hat die Auslegungen des Stücks besonders erschwert, welches bald mit der Betonung auf den komischen bald auf den tragischen Aspekten inszeniert wird ${ }^{3}$. Die hier interessierende Fragestellung liegt jenseits der Beschäftigung mit der komischen oder tragischen Wirkung des Stücks: Molières Misanthrope ist ein Theaterstück über die Formen der indirekten Kommunikation und reflektiert die Frage, welche Situationen diese erfordern und ob die indirekte Kommunikation überhaupt zu umgehen ist.

Das Stück ist geradezu systematisch aufgebaut: Die Komödie wird durch ein langes Gespräch der beiden Freunde Alceste und Philinte eröffnet, ein ,entretien familier', der die größtmögliche Offenheit in der zeitgenössischen Konversation erlaubt. In dieser Eingangskonversation werden die beiden konträren Positionen abgesteckt, die das Stück in seinem Verlauf durchspielt und reflektiert. Die zentrale Frage lautet, ob man stets ehrlich und offen sprechen soll, oder ob die gesellschaftlichen Gebote der höflichen Gefälligkeit (,complaisance') indirekte Formen der Kommunikation erfordern und die allzu große Offenheit verbieten. Dabei geht Molière wie bei einer mittelalterlichen, scholastischen Untersuchung (,quaestio`) vor: Zunächst werden im Eingangsgespräch die Positionen vorgestellt und es werden die Argumente zusammengetragen, die für und gegen die jeweilige Position sprechen. Alsdann zeigt sich im Laufe des Stücks, dass weder die eine noch die andere Position in ihrer eingangs vertretenden Form zu halten ist. Dabei verfährt Molière nach dem Prinzip der Steigerung, die Positionen in einer immer groteskeres Licht zu rücken. In seiner literarischen Behandlung der ,quaestio“ lässt er, wie man das bereits aus dem Partimen der mittelalterlichen Liebesdichtung kennt ${ }^{4}$, die letztendliche Beantwortung der Fragen offen und überlässt es dem Zuschauer, die freigesetzten Reflexionen für sich zu bewerten.

3 Vgl. dazu Peter Schunck, „Zur Wirkungsgeschichte des Misanthrope“, Germanisch-Romanische-Monatsschrift Bd. 21/1971, S. 1-15, wieder abgedruckt in: Renate Baader (Hrsg.), Molière (Wege der Forschung. 261), Darmstadt, Wissenschaftliche Buchgesellschaft, 1980, S. 384-405.

4 Vgl. dazu das Buch von Sebastian Neumeister, Das Spiel mit der höfischen Liebe. Das Altprovenzalische Partimen (Beihefte zu Poetica. 5), München, Fink, 1969. 


\subsection{Aufrichtiges oder verdecktes Sprechen? Das Gespräch zwischen Alceste und Philinthe zu Beginn des Misanthrope}

Der Misanthrop Alceste macht seinem Freund Philinte Vorhaltungen wegen dessen allgemeiner Gefälligkeit (,complaisance') gegenüber Dritten. Die ,complaisance، steht hier für die indirekten Formen des Sprechens aus Höflichkeit. Alceste sieht in diesem Verhalten eine Heuchelei und gibt gleich zu Beginn sein eigenes Credo preis:

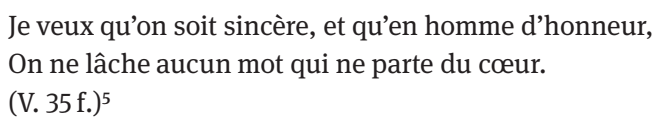

Der Protagonist offenbart ein Verständnis vom , honnête homme‘, welches dem der Epoche diametral zuwider läuft: Der ,honnête homme“ muss seiner Meinung nach stets offen und aufrichtig sprechen. Damit eröffnet Alceste eine Debatte, die das gesamte folgende Jahrhundert, die Aufklärung, prägen wird, und deren Aporien er nicht im Mindesten erahnt. Alceste ist jede Form des Sprechens verhasst, die in verdeckter Form erfolgt. Der Ehrenmann folgt - so der Protagonist des Stücks der Sprache seines Herzens. Wenig später wird dies von ihm präzisiert:

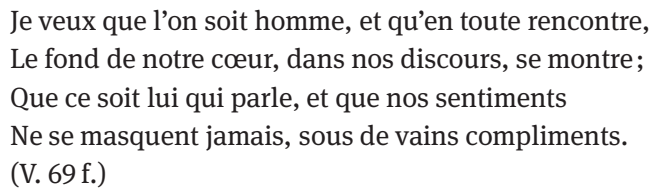

Nun haben wir es bei Alceste noch nicht mit jener Forderung nach Aufrichtigkeit zu tun, die die moralischen Qualitäten des sprechenden Subjekts unter Beweis stellt. Seine Forderung nach der Sprache des Herzens ist noch nicht die der Aufklärung, die in der Übermittlung der - im 18. Jahrhundert als aufrichtig geltenden - Gefühle des Sprechers den Ausweis seiner Integrität zu erkennen vermeint. „Le mot qui part du cœur“ bedeutet bei Alceste nichts anderes, als sich ohne ausschweifende Höflichkeitsfloskeln der direkten Rede zu bedienen. Seine ,sincérité‘ ist - so versteht das 17. Jahrhundert den Begriff - eine Äußerung ohne ,dissimulation'. Eine solche bedingungslose Aufrichtigkeit wird in der Epoche jedoch

5 Zitierte Ausgabe: Moliere, Euvres complètes, 2 Bde., hrsg. von Georges Couton (Bibliothèque de la Pléiade. 8. 9), Paris, Gallimard, 1971, Bd. 2, S. 141-218, hier: S. 143. Die Versangaben im laufenden Text beziehen sich auf diese Ausgabe. 
als Verstoß gegen die Geselligkeitsregeln gesehen, und genau davon handelt der Streit von Alceste mit seinem Freund Philinte zu Beginn des Stücks wie auch der weitere Verlauf der gesamten Komödie.

Alceste wirft Philinte vor, sich aus Höflichkeit völlig unbekannten Dritten gegenüber allzu gefällig zu verhalten:

Je vous vois accabler un homme de caresses,

Et témoigner, pour lui, les dernières tendresses;

De protestations, d'offres, et de serments,

Vous chargez la fureur de vos embrassements:

Et quand je vous demande après, quel est cet homme,

À peine pouvez-vous dire comme il se nomme,

[...] (V. 17-22)

Für Alceste gleicht ein solches Verhalten der Prostitution (V. 54). Er vermag in der ,complaisance' nur eine oberflächliche, formelle zwischenmenschliche Beziehung zu erkennen, welche alle Unterschiede einebnet und den Wert des Einzelnen herabsetzt. Dieser Gleichmacherei widersetzt er sich und sie ist es, die ihn letztlich zum Misanthropen hat werden lassen:

Je refuse d'un cœur la vaste complaisance,

Qui ne fait de mérite aucune différence:

Je veux qu'on me distingue, et pour le trancher net, L'ami du genre humain n'est point du tout mon fait.

(V. 61-54)

Demgegenüber betont Philinte den Wert des indirekten Sprechens, den die Höflichkeit und die ,bienséance‘ erfordert. Für ihn gilt, eine höfliche Ansprache auch mit gleicher Münze zu bezahlen:

Lorsqu'un homme vous vient embrasser avec joie,

Il faut bien le payer de la même monnoie,

Répondre, comme on peut, à ses empressements,

Et rendre offre pour offre, et serments pour serments.

(V. 37-40)

Auffällig ist bereits hier die Begründung des höflichen Umgangs und der höflichen Kommunikation mit ökonomischen Kategorien (,il faut bien payer“ / „rendre offre pour offre“), eine Sprache, der sich Molière im weiteren Verlauf bei der Entlarvung des indirekten Sprechens als Täuschung des Konversationspartners aus Eigennutz mehrfach bedient und somit den Nutzcharakter der ,complaisance' freilegt. Offenheit wird von Philinte als Verstoß gegen die ,bienséance‘ gewertet: 
Serait-il à propos, et de la bienséance,

De dire à mille gens tout ce que d'eux, on pense?

(V. $77 \mathrm{f.})$

Die Offenheit sei oftmals einer der großen ,faux pas‘ des ,honnête homme‘ und des ,bel esprit‘. Sie führe dazu, dass der Sprecher sich lächerlich (,ridicule“) mache und - so beschreiben es auch die zahlreichen Traktate höfischen Verhaltens gesellschaftlich diskreditiert sei. Deshalb rät Philinthe Alceste mit Nachdruck, davon abzulassen:

\footnotetext{
Il est bien des endroits, où la pleine franchise

Deviendrait ridicule, et serait peu premise;

(V. $73 \mathrm{f}$.

Non, tout de bon, quittez toutes ces incartades,

Le monde, par vos soins, ne se changera pas;

Et puisque la franchise a, pour vous, tant d'appas,

Je vous dirai tout franc, que cette maladie,

Partout où vous allez, donne la comédie,

Et qu'un si grand courroux contre les mœurs du temps,

Vous tourne en ridicule auprès de bien des gens.
}

(V. 102-108)

Grundsätzlich gilt - dabei beruft sich Philinte auf die menschliche Natur - dass das menschliche Verhalten anpassungsfähig und geschmeidig bleiben soll und dass die „vertu traitable“ niemals den Maßstäben einer rigiden Vernunft unterworfen werden darf. Philinte greift damit neustoische Positionen an, die aus der in der Epoche viel diskutierten stoischen Tugendlehre stammen. Er tritt für eine, zeitgemäße Weisheit‘ ein, die vor allem danach trachtet, andere nicht zu belehren:

Mon Dieu, des mœurs du temps, mettons-nous moins en peine,

Et faisons un peu grâce à la nature humaine;

Ne l'examinons point dans la grande rigueur,

Et voyons ses défauts, avec quelque douceur.

Il faut, parmi le monde, une vertu traitable,

À force de sagesse on peut être blâmable,

La parfaite raison fuit toute extrémité,

Et veut que l'on soit sage avec sobriété.

Cette grande raideur des vertus des vieux âges,

Heurte trop notre siècle, et les communs usages,

Elle veut aux mortels, trop de perfection,

Il faut fléchir au temps, sans obstination;

Et c'est une folie, à nulle autre, seconde,

De vouloir se mêler de corriger le monde.

(V. 145-158) 
Die von Philinte geforderte und praktizierte ,complaisance' orientiert sich am IstZustand der Gesellschaft. Dieser ist, wie es der Theoretiker des absolutistischen Machtstaats Hobbes formuliert hatte, ein ,bellum omnium contra omnes'. Sogar Hobbes berühmtes Diktum vom ,homo homini lupus est' taucht in den Überlegungen Philintes auf:

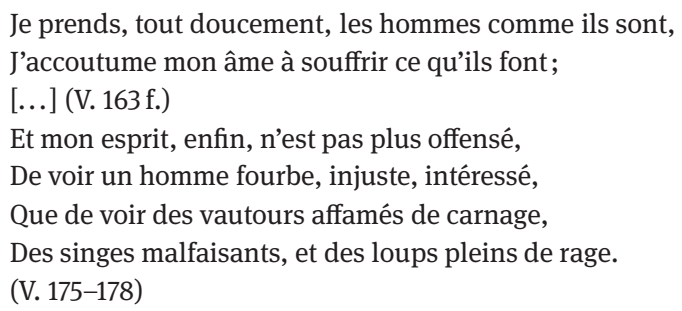

Der weitere Verlauf des Misanthrope führt nun auf der einen Seite Beispiele dieser ,complaisance' vor, die deren moralische und ästhetische Seite als Tarnung entlarven und in vollem Umfang hervortreten lassen, wie sehr die indirekten Formen des Sprechens mit ihren Formeln der ,complaisance“ und ,politesse“ der Eigenliebe (,amour propre') und dem Nutzen des Sprechers dienen. Auf der anderen Seite wird jedoch auch Alcestes Haltung unbedingter Offenheit einer Prüfung unterzogen und letztendlich als nicht haltbar entlarvt.

\subsection{Indirektes Sprechen und die Sprache der Literatur: Alcestes Kritik am Sonett des Oronte}

Der erste Akt des Stücks steht mit dem Gespräch zwischen Alceste und Philinte sowie dem zwischen Oronte und Alceste auch im Zeichen des Themas ,Freundschaft'. Oronte, ein aristokratischer Verehrer Célimènes, um die auch Alceste wirbt, sucht sich mit einer schmeichlerischen Ansprache zum Schein der Freundschaft des Protagonisten zu versichern:

J'ai monté, pour vous dire, et d'un cœur véritable, Que j'ai conçu pour vous, une estime incroyable; Et que, depuis longtemps, cette estime m'a mis Dans un ardent désir d'être de vos amis.

Oui, mon cœur, au mérite, aime à rendre justice, et qu'un nœud d'amitié nous unisse [...]

(V. 253-258)

Nachdem Alceste versucht, mit dem Argument, einen neuen Freund erst besser kennen lernen zu müssen, das Ansinnen aufzuschieben, um es letztlich ablehnen 
zu können, erhöht Oronte den Druck mit dem Angebot, er könne dem Protagonisten dank seiner Beziehungen bei Hof manche Tür öffnen:

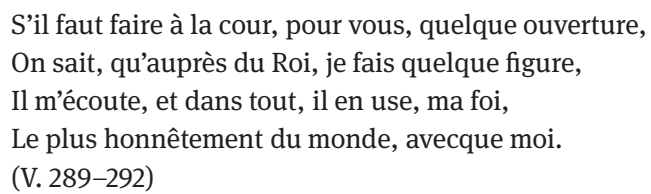

Mit diesem Argument für die Freundschaft hat Oronte die hierarchischen Relationen zwischen den beiden Sprechern umgekehrt: Nach der zunächst unterwürfigen indirekten Ansprache gegenüber Alceste, den er angeblich über die Maßen schätzt (,estime incroyable“), versetzt sich Oronte mit dem Angebot, ihm dank seiner Beziehungen wichtige Kontakte bei Hof zu verschaffen, auf einen Schlag in eine Alceste überlegene Rolle. Letzten Endes beabsichtigt er, sich Vorteile beim Werben um die Gunst der Célimène zu verschaffen und sich die Bewunderung eines von ihm verfassten Sonetts durch Alceste einzuholen. Als er dann Alceste sein Sonett vorträgt, wird erneut das zentrale Thema der Komödie ins Spiel gebracht:

\footnotetext{
L'espoir, il est vrai, nous soulage,

Et nous berce un temps, notre ennui :

Mais, Philis, le triste avantage,

Lorsque rien ne marche après lui !

Vous eûtes de la complaisance,

Mais vous en deviez moins avoir;

Et ne vous pas mettre en dépense,

Pour ne me donner que l'espoir.

S'il faut qu'une attente éternelle

Pousse à bout, l'ardeur de mon zèle,

Le trépas sera mon recours.

Vos soins ne m'en peuvent distraire;

Belle Philis, on désespère,

Alors qu'on espère toujours.
}

Die Literatur zum Misanthrope hat stets behauptet, das Thema dieses Sonetts sei die Hoffnung ${ }^{6}$. Im Stil der petrarkistischen Dichtung, die in immer neuen Varianten die widersprüchlichen Leidenschaften der Liebenden (,contrari affetti`) durch-

6 Vgl. dazu stellvertretend Frank W. Lindsay, „Alceste and the Sonnet“, The French Review Bd. 28/1955, H. 5, S. 395-402, Richard J. Wayne, „Contradiction in the Sonnet Scene of Le Misanthrope“, in: Esprit créateur Bd. 15/1975, H. 1-2, S. 154-163. Vgl. auch Norman Henfrey, „Towards a View of Molière's Misanthrope: The Sonnet Scene Reconsidered”, The Cambridge Quaterly Bd. 18/1989, H. 2, S. 160-186. 
spielt, dreht sich die Reflexion hier in der Tat um die gegensätzlichen Befindlichkeiten von Hoffnung (,espoir“, V. 1) und Niedergeschlagenheit („ennui“, V. 2). Schaut man jedoch etwas genauer hin, knüpft das Sonett eher an das Thema des ersten Akts an: die „complaisance“ (V. 5). Célimène wird von Alceste wie von Oronte gleichermaßen verehrt und hält beide durch indirekte und uneindeutige Äußerungen hin. Ausgerechnet Oronte, der sich mit seinen Formeln der ,complaisance' als ein auffälliger Vertreter der Verstellung erweist und dessen als ,politesse' getarnte Annäherungen an den Protagonisten ausschließlich auf den eigenen Vorteil beim Werben um Célimène ausgerichtet sind, dichtet ein Sonett, in dem ,Phillis“ - gemeint ist natürlich Célimène - aufgefordert wird, weniger ,complaisance“ zu praktizieren (,vous en deviez moins avoir“, V. 6), da der Sprecher ansonsten dem Tod verfallen würde („Le trépas sera mon recours“, V. 11). Oronte sieht sich somit in seinem Sonett als Opfer der ,complaisance' seiner Dame. Und so bezieht sich denn auch die offen vorgetragene Kritik des Alcestes am Sonett, zu der Oronte ihn hartnäckig drängt, auf die ,complaisance‘ des Gedichts:

\footnotetext{
Franchement, il est bon à mettre au cabinet;

Vous vous êtes réglé sur de méchants modèles,

Et vos expressions ne sont point naturelles.

Qu'est-ce que nous berce un temps, notre ennui,

Et que rien ne marche après lui?

Que ne vous pas mettre en dépense,

Pour ne me donner que l'espoir?

Et que Philis, on désespère,

Alors qu'on espère toujours?

Ce style figuré, dont on fait vanité,

Sort du bon caractère, et de la vérité;

Ce n'est que jeu de mots, qu'affectation pure,

Et ce n'est point ainsi, que parle la nature.

Le méchant goût du siècle, en cela, me fait peur,

[...] (V. 376-389; Hervorhebungen von Vf.)
}

Durch Orontes Gedicht über die ,complaisance“ wird gezeigt, inwieweit auch die zeitgenössische Literatur in die Reflexion über die Formen des indirekten Sprechens eingebunden ist. Literatur ist per se eine Form der indirekten Äußerung. Alceste kritisiert am Beispiel einiger Floskeln des Gedichts den „style figuré“, in dem er nur Wortspiele und Affektiertheit zu erkennen vermag. Diese indirekte Form des Sprechens ist für ihn Zeichen der Eitelkeit ihres Verfassers. Alceste kritisiert somit das, was die literarische Sprache ausmacht als unnatürlich und als Angst erzeugenden schlechten Geschmack der Zeit. Reden in indirekter Form - und damit ist vor allem die alltägliche Rede der Epoche im Zeichen der ,complaisance gemeint - ist somit für ihn grundsätzlich literarische Rede. Diese Offenheit bringt 
ihm seitens des Oronte eine Aufforderung zum Duell ein, die letztlich vor einem Schiedsgericht verhandelt wird, da Richelieu bekanntlich das Duell verboten hatte. Der weitere Verlauf des Stücks bringt nun in gesteigerter Form Beispiele des indirekten Sprechens ins Spiel.

\subsection{Das Scheitern des indirekten Sprechens im Zeichen der ,complaisance': die ,scène des portraits` und das Gespräch zwischen Célimène und Arsinoé im zweiten und dritten Akt des Misanthrope}

Der zweite Akt des Stücks erweitert den Blickwinkel von der Freundschaft auf das Thema der Liebe und des Liebeswerbens. Er beginnt mit einem längeren Gespräch zwischen Alceste und Célimène, der der Protagonist vorwirft, mit ihrer ,complaisance' allzu viele Erwartungen bei allzu vielen Verehrern zu erwecken. Célimène, die die jungen sie umschwärmenden Aristokraten hinhält, macht keinen Hehl daraus, dass ihre uneindeutigen Ansprachen an die Verehrer allein der Verfolgung ihrer Interessen dienen. Auf die Frage Alcestes, warum sie Clitandre mit ihren ,complaisances' bedenkt, antwortet sie:

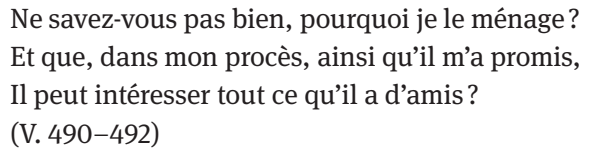

Berühmt geworden ist die Szene der ,portraits', vor allem weil es sich dabei um eine Modeerscheinung der zeitgenössischen Salonkonversation handelt. Célimène wird jeweils der Name eines zumeist männlichen Aristokraten genannt, worauf sie eine kurze Charakteristik der Person gibt. Auf diese Weise steht sie nicht nur als Einladende im Mittelpunkt der Konversation ihres Salons; sie hat auch eine Schiedsrichterfunktion und kann ihre geradezu meisterliche Kunst der Personendarstellung zum Besten geben. Dabei steht die gesamte Szene unter dem Aspekt der ,maldisance': Bei jeder Person wird ein auffälliges Fehlverhalten (,défaut') thematisiert, jedoch in einer Form, die vermeidet, allzu sehr den Tatbestand einer beleidigenden Beschreibung zu erfüllen. Einige wenige Beispiele aus der lange Reihe der Porträts können dies verdeutlichen:

CLITANDRE

Timante encor, madame, est un bon caractère.

CÉLIMÈNE

C’est de la tête aux pieds un homme tout mystère, 
qui vous jette en passant un coup d'œil égaré, et, sans aucune affaire, est toujours affairé.

Tout ce qu'il vous débite en grimaces abonde;

à force de façons, il assomme le monde;

sans cesse il a, tout bas, pour rompre l'entretien,

un secret à vous dire, et ce secret n'est rien;

(V. 585-612)

Timante ist ein im Grunde uninteressanter Mensch, der sich eine Aura des Geheimnisvollen und Undurchdringlichen gibt, um als interessant zu erscheinen. Insofern ist er eine ,personificatio“ eines bestimmten Typs des indirekten Sprechens: der verklausulierten Rede, um deren geringen Gehalt zu überdecken. Auf diese Weise werden nach und nach diverse Typen ins Spiel gebracht und mit ihren Fehlern entlarvt: „Cléonte, le ridicule achevé“ (V. 567 f.), der sich durch seine ostentativ zur Schau gestellte Extravaganz überall lächerlich macht, „Damon, le raisonneur“ (V. 577), der sein Gegenüber zwingt, seinen unerträglich weitschweifigen Ausführungen zuzuhören, „Géralde, l'ennuyeux conteur“ (V. 594 f.), der seine langweiligen Erzählungen durch die Nennung hochgestellter Persönlichkeiten zu nobilitieren versucht, „Bélise, „le pauvre esprit de femme“ (V. 604 f.), die mit ihrer sterilen Ausdrucksweise („la stérilité de son expression“, V. 607) das genaue Gegenstück zu Célimène darstellt, „Adraste, un homme gonflé de soi-même“ (V. 618), der stets gegen den Hof wettert, „Damis, [qui] veut avoir trop d'esprit“ (V. 634), dessen Geschmack grundsätzlich nichts standhalten kann („Rien ne touche son goût, tant il est difficile;“, V. 638). All diese Formen des Fehlverhaltens fallen in den Bereich der Konversation und der Sprache, selbst dort wo es vom ,jeune Cléonthe“ (V. 623) heißt, er rühme sich seines Kochs, obwohl dieser die Speisen verderbe (,il gâte, à mon goût, tous les repas qu'il donne“, V. 630), stehen doch das Menü und die Zubereitung der Speisen traditionell allegorisch für die Rede und die Konversation des Gastgebers.

Sprechen aus ,complaisance' wird auch im Folgenden als heuchlerisch entlarvt, wenn es im dritten Akt zu einem Dialog zweier weiterer Rivalen beim Werben um die Gunst der Célimène, Clitandre und Acaste, kommt. Als Letzterer seinem Rivalen die lange Reihe seiner Vorzüge vor Augen führt, die ihm bei den Damen Chancen einbringen, verfällt er ungewollt und von ihm unbemerkt in eine entlarvende ökonomische Sprache:

\footnotetext{
Mais les gens de mon air, Marquis, ne sont pas faits, Pour aimer à crédit, et faire tous les frais.

Quelque rare que soit le mérite des belles, Je pense, Dieu merci, qu'on vaut son prix, comme elles; Que pour se faire honneur d'un cœur comme le mien, Ce n'est pas la raison qu'il ne leur coûte rien;
} 
Et qu'au moins, à tout mettre en de justes balances,

Il faut, qu'à frais communs, se fassent les avances.

(V. 815-822; Hervorhebungen von Vf.7)

Die Sprache über die Damen des jungen Aristokraten, deren dahinter liegendes Denken sich selbst entlarvt, wird nur noch übertroffen durch die der weiblichen Protagonistinnen. Gipfelpunkt ist der Dialog zwischen Célimène und Arsinoé am Ende des dritten $\mathrm{Akts}^{8}$. Nach anfänglichem indirekten Sprechen mündet diese Konversation alsbald in eine offene Auseinandersetzung, in der die Schleier der verdeckten Rede fallen gelassen werden. Molière zeigt mit dieser Szene, dass die Rede im Zeichen von ,bienséance‘, ,politesse‘ und ,complaisance‘ nicht immer durchzuhalten ist.

Arsinoé nähert sich der Protagonistin und Herrin des Salons als vermeintliche Freundin, die Célimène aus Ehrerbietung („honneur“) und Wohlanständigkeit („bienséance“) anvertraut, wie man anderweit in Gesellschaft schlecht über sie gesprochen habe. In Wirklichkeit will Arsinoé jedoch Alceste umwerben und ihm einen Brief übergeben, den Célimène an Oronte geschrieben hat, um auf diese Weise den Beweis der Untreue von Célimène zu liefern.

[...] comme il n'en est point de plus grande importance

Que celles de l'honneur, et de la bienséance, Je viens, par un avis qui touche votre honneur, Témoigner l'amitié que, pour vous, a mon cœur. Hier, j'étais chez des gens, de vertu singulière, Où, sur vous, du discours, on tourna la matière;

Et là, votre conduite, avec ses grands éclats, eut le malheur, qu'on ne la loua pas.

(V. 881-888)

Kritisiert wurde in der Gesellschaft jene ,complaisance', die Célimène nach Meinung der Konversationsteilnehmer allzu vielen zukommen lässt:

Cette foule de gens, dont vous souffrez visite,

Votre galanterie, et les bruits qu'elle excite,

Trouvèrent des censeurs plus qu'il n'aurait fallu,

Et bien plus rigoureux que je n'eusse voulu.

Vous pouvez bien penser quel parti je sus prendre;

(V. 889-893)

7 Darauf verweist auch Renée-Claude Lorimier, „Le Secret dans Le Misanthrope de Molière: agrément courtois ou arme politique?”, Études littéraires Bd. 28/1995, H. 2, S. 97-106, hier: S. 101 f. mit Anm. 7 .

8 Zur Figur der Arsinoé vgl. James L. Sheperd, III, „Arsinoé as Puppeteer”, The French Review Bd. 42/1968, H. 2, S. 262-271. 
Arsinoé behauptet zunächst, dass sie Célimène verteidigt habe, erklärt dann jedoch heuchlerisch, sie sei letztlich in die Enge getrieben worden und habe ein Stück weit den Kritikern zustimmen müssen:

\footnotetext{
Vous pouvez bien penser quel parti je sus prendre;

Je fis ce que je pus, pour vous pouvoir défendre, Je vous excusai fort sur votre intention, Et voulus, de votre âme, être la caution.

Mais vous savez qu'il est des choses dans la vie, Qu'on ne peut excuser, quoiqu'on en ait envie; Et je me vis contrainte à demeurer d'accord, Que l'air dont vous viviez, vous faisait un peu tort. (V. 893-900)
}

Célimène kontert diese immer offensichtlichere und offener geführte Attacke, indem sie ihrerseits berichtet, wie anderweit von der so prüden Arsinoé gesprochen werde. In dem als verbales Duell angelegten Gespräch entlarvt Célimène auf eine sprachlich unnachahmliche Art ihre sich als Freundin tarnende Rivalin:

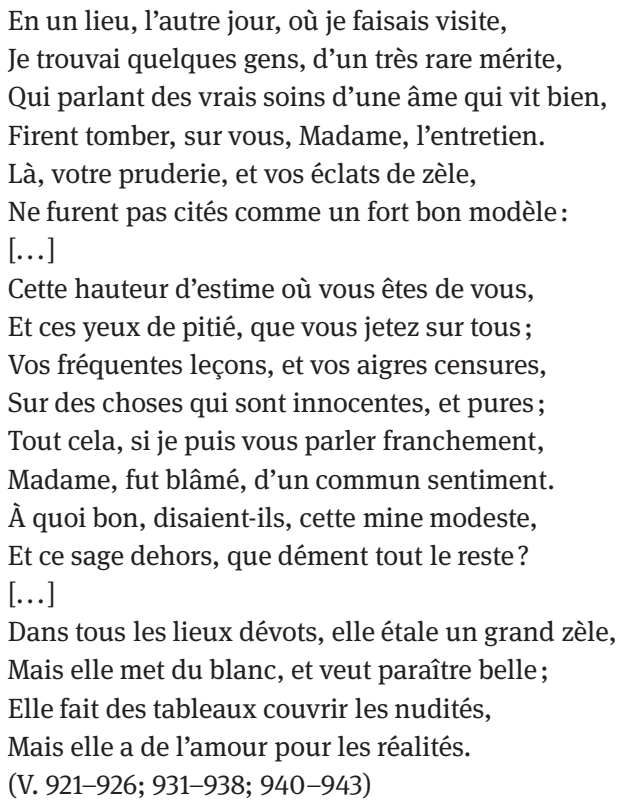

Die Berufung auf die „gens d'un très rare mérite” sucht der Argumentation eingangs die nötige Autorität zu verleihen. Die gesamte Rede ist eine Entlarvung der verdeckten Sprache und Körpersprache der Rivalin mit dem Ziel, sie als moralisch 
fragwürdig darzustellen. Der Gestus ist der der zunehmenden Enthüllung: Arsinoés Sprache ist die des ,paraître‘ (V. 941), hinter der letztlich die ,nudités“ (V. 942) aufscheinen. Nach außen hin gibt sich Arsinoé prüde, dahinter ist sie jedoch der Realität zugetan („l’amour pour les réalités“, V. 943), wobei die Konnotation, die die Floskel auslöst, lautet: Arsinoé schätzt die ,réalités de l'amour‘. Arsinoé vermeint zu erkennen, dass ihre Offenheit Célimène beleidigt hat: „Madame, je vois bien [...] / Que mon sincère avis vous a blessée au cœur“ (V. 963f). Célimène erklärt ihr jedoch ganz pragmatisch, dass man solch offene Äußerungen zum beiderseitigen Vorteil handhaben solle, um der generellen Verblendung durch das indirekte Sprechen zu entgehen. Sie dementiert damit ihre eigenen Regeln, die normalerweise auf eine ,complaisance‘ ausgerichtet sind und den Gesprächspartner im Unklaren lassen:

\footnotetext{
Au contraire, Madame, et si l'on était sage, Ces avis mutuels seraient mis en usage;

On détruirait, par là, traitant de bonne foi, Ce grand aveuglement, où chacun est pour soi.

Célimène macht Arsinoé klar, dass es für das menschliche Verhalten keine festen Regeln geben kann, sondern dass jede Situation Regeln eigener Art erfordert. Und dies gilt auch für das Sprechen. Sie ermöglicht damit Arsinoé, dem Streit unter Wahrung ihres Gesichts zu entkommen. Gleichzeitig wird deutlich, wie wenig die Position indirekter Kommunikation durchgehend zu halten ist:

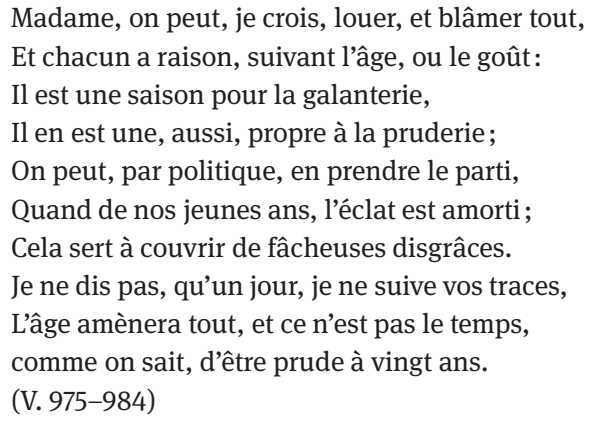




\subsection{Das Scheitern der ,sincérité‘ im Fall des Alceste}

Was für die Übertreibung der ,complaisance‘ gilt, gilt mehr noch für Alceste und seine Offenheit. Das Stück liefert gleich zu Beginn ein Beispiel für diesen Befund: Der Eingang der Komödie erfolgt abrupt in medias res, ohne dass die Personen in der üblichen Weise eingeführt werden. Es beginnt mit einer schroffen Gesprächsverweigerung, die zeigt, was Alcestes' Offenheit im sozialen Kontext bedeutet: die Zerstörung jeglicher Geselligkeit. Molière führt auf diese Weise vor, wie eine schroffe ,Aufrichtigkeit‘ aussieht und was sie bewirkt, ohne dass er das Thema des Stücks bereits angesprochen hat:

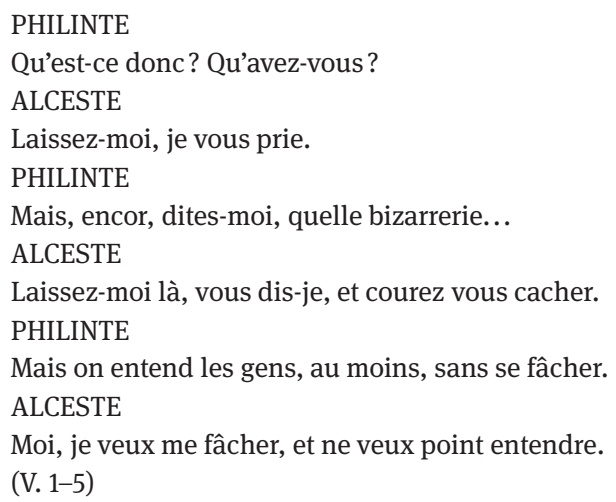

Der Misanthrope endet auf die gleiche Weise. Mit geradezu beleidigenden Worten erklärt der Protagonist Célimène, er wolle ihre Verbrechen (also ihre ,complaisances $^{`}$ ) als Jugendsünde entschuldigen, vorausgesetzt sie lasse die Gesellschaft hinter sich und gehe mit ihm in die ,retraite':

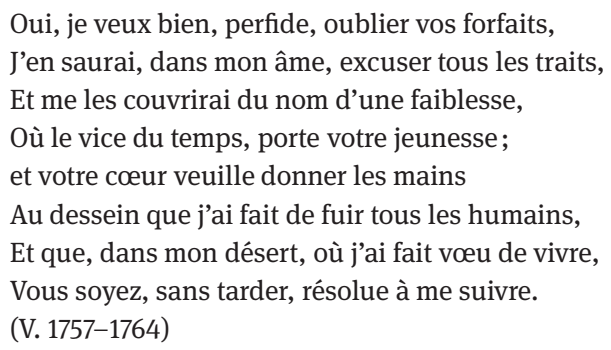

Da sie sich weigert, sich „mit ihm in der Wüste zu begraben“ (,dans votre désert aller m'ensevelir“, V. 1770) zieht sich der Protagonist unversöhnt und allein zurück: 
Je vais sortir d'un gouffre où triomphent les vices;

Et chercher sur la terre, un endroit écarté, homme d'honneur, on ait la liberté.

(V. 1804-1806)

Das Stück führt auch vor, dass Alceste seine Einstellung nicht durchhalten kann. Daraus resultiert eine „Komik des inkonsequenten Handelns“9. Elisabeth Schulze-Witzenrath hat diese Inkonsequenzen im Verhalten des Protagonisten detailliert beleuchtet. Alcestes Wunsch, durch seine Offenheit als Individuum gewürdigt zu werden (,Je veux qu'on me distingue [...]“, V. 63), steht im Widerspruch zu seiner trotzigen Reaktion auf Philintes Mahnung, er mache sich überall lächerlich:

Tant mieux, morbleu, tant mieux, c'est ce que je demande,

Ce m'est un fort bon signe, et ma joie en est grande:

[...] (V. 109f.)

In Wirklichkeit ist auch er ein Mensch bestehend aus Vernunft und Gefühl und somit eine widersprüchliche Figur:

II est vrai : ma raison me le dit chaque jour;

Mais la raison n'est pas ce qui règle l'amour.

(V. $247 \mathrm{f}$.

So liegt denn auch ein Widerspruch darin, dass ausgerechnet er als Vertreter einer bedingungslosen Offenheit Célimène zugetan ist, die sich ihrerseits ganz dem Prinzip der Verstellung verpflichtet fühlt. Mit einer ironischen Bemerkung hält ihm Philinte im Eingangsgespräch diesen Widerspruch vor:

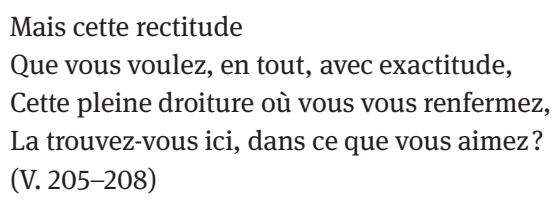

Besonders auffällig ist dieser Widerspruch, wenn Alceste durch die Situation bedingt seinerseits dazu gedrängt wird, sich der ,complaisance' und damit der indirekten Formen des Sprechens zu bedienen, um der direkten Konfrontation auszuweichen. Schulze-Witzenrath hat gezeigt, wie Molière dies im Fall der Konver-

9 Vgl. Elisabeth Schulze-Witzenrath, „Konversation und Komik in Molières Misanthrope. Warum sich eine Komödie des 17. Jahrhunderts heute nicht mehr erfolgreich aufführen lässt“, Poetica Bd. 27/1995, S. 273-313, hier: S. 285. 
sation zwischen Oronte und Alceste nach und nach entwickelt: Als ihm Oronte schmeichlerisch die Freundschaft anträgt, beruft sich Alceste als Vertreter der Offenheit ausgerechnet auf das Geheimnisvolle freundschaftlicher Verbindungen, welches es erst zu entdecken gelte („L'amitié demande un peu plus de mystère [...]“, V. 278). Seine lehrhaften Sätze über die Freundschaft treffen mit Oronte auf einen Gesprächspartner, der auf einen schmeichlerischen Austausch von Höflichkeiten aus ist, jedoch keinerlei ,conversation sérieuse' über die Freundschaft im Allgemeinen erwartet und entsprechend verblüfft reagiert: „Parbleu? c'est làdessus parler en homme sage [... ]“ (V. 285) ${ }^{10}$. Und schon gar nicht erwartet Oronte, dass Alceste eine ehrliche und offene Kritik seines Sonetts abliefert, obwohl er aus Höflichkeit bekundet, dessen Offenheit zu schätzen und eine offene Kritik zu wollen. Als Alceste dann zu seiner Kritik gedrängt wird, bringt er es zunächst nicht fertig, offen zu sprechen. Er erfindet einen Fall, in dem er jemand geraten hat, seine Verse nicht zu veröffentlichen (V. 341-350).

Das Stück ist somit ein Beleg für die von vielen Traktaten geforderte ,souplesse' des Verhaltens. Weder die übermäßige ,complaisance“ noch die schroffe, sincérité des Alceste bleiben von Molières Gespött verschont. Die Figuren werden jeweils in ein Verhalten getrieben, welches nicht ihren eigenen Regeln und schon gar nicht denen der Gesellschaft entspricht. Molière führt nicht nur vor, wie Übertreibungen des Verhaltens aussehen. Er führt auch vor, wie die Figuren situationsbedingt gezwungen sind, die sich selbst verordneten Regeln aufzugeben, z. B. wenn Célimène und Arsinoé die Regeln des indirekten Sprechens zugunsten einer immer schrofferen und verletzenden Offenheit durchbrechen und Alceste im Gespräch mit Oronte in die ,complaisance' getrieben wird, die er verabscheut. Allein Philintes mäßigende Worte an den Protagonisten können als Maßstab eines vernünftigen, $d$. h. geschmeidigen Verhaltens der Epoche dienen, an dem die Übertreibungen gemessen werden können. Dies wird zu Beginn im Gespräch mit dem Misanthrope offenbar und tritt am Ende des Stücks erneut zutage. Komik entsteht somit durch die Abweichung vom normgerechten Verhalten, und dies triff die indirekte Form des Sprechens gleichermaßen wie die ohnehin weitgehend verpönte Form der Offenheit.

10 Vgl. Schulze-Witzenrath, „Konversation und Komik“, S. 293. 